Full Citation - Chamberlain, A. et al. 'Locating Experience: touring a pervasive performance', Personal Ubiquitous Computing Journal, Volume 15 Number 7 . Springer Verlag. ACM Library. DOI: 10.1007/ s00779-010-0351-3. pp. 717-730

Full Download - http://dl.acm.org/citation.cfm?id=2039114

\title{
Locating Experience: Touring a Pervasive Performance
}

Alan Chamberlain, *Leif Opperman, Martin Flintham, Steve Benford, Peter Tolmie, **Matt Adams, **Ju Row Farr, **Nick Tandavanitj, Joe Marshall, Tom Rodden.

$\begin{array}{ll}\text { Mixed Reality Laboratory } & \text { *Fraunhofer Institute for Applied } \\ \text { University of Nottingham } & \text { Information Technology FIT } \\ \text { Nottingham, NG8 1BB, } & \text { Schloss Birlinghoven } \\ \text { UK } & \text { 53754 Sankt Augustin, Germany } \\ \text { \{azc\}@cs.nott.ac.uk } & \text { \{eif.oppermann\}@fit.fraunhofer.de } \\ \text { Tel: +44 115 8466530 } & \text { Tel: +492241 142724 } \\ \text { Fax: +44 115 8466416 } & \text { Fax: }+492241142080 \\ \text { URL: www.mrl.nott.ac.uk } & \text { URL: http://www.fit.fraunhofer.de }\end{array}$

\author{
**Bast Theory \\ Unit 5, 20 Wellington Road \\ Brighton, BN41 1DN, UK \\ \{matt\}@blasttheory.ac.uk \\ Tel: +44 1273413455 \\ URL: www.blasttheory.ac.uk
}

\begin{abstract}
Touring location-based experiences is challenging, as both content and underlying location-services must be adapted to each new setting. A study of a touring performance called Rider Spoke as it visited three different cities reveals how professional artists developed a novel approach to these challenges in which users drove the co-evolution of content and the underlying location-service as they explored each new city. We show how the artists iteratively developed filtering, survey, visualization and simulation tools and processes to enable them to tune the experience to the local characteristics of each city. Our study reveals how by paying attention to both content and infrastructure issues in tandem the artists were able to create a powerful user experience that has since toured to many different cities.
\end{abstract}

Keywords: Location-based performance, cycling, adaptation, Wi-Fi fingerprinting, seams, user generated content, Rider Spoke.

\section{Introduction}

From tours and guides, through games and performances, to educational field trips, there is a growing interest in how location-based technologies can deliver engaging new forms of leisure, entertainment and learning. Early explorations hav ${ }^{1}$ e demonstrated a variety of ways in which such experiences can combine digital media with physical settings to create exciting new experiences. Guides and tours have attached digital media to historic sites in order to enhance understanding $[1,7,11]$. Computer games have

\footnotetext{
${ }^{1}$ This paper expands upon the paper Lessons from Touring a Location-Based Experience, presented at Pervasive 2011 in terms of its ethnographic content.
} 
been overlaid on real cities, demanding physical engagement from players as they chase one another through the streets $[4,6,10,17,21]$. Other experiences have drawn on the pleasure of physical exploration, either to find digital resources $[2,16]$ or to find physical resources as in the popular pastime of Geocaching [18]. Educational experiences have engaged learners in simulations that appear to be overlaid on the real world [5] and have underpinned field trips to sites of special interest [19,23]. Location-based technologies have also enhanced Live Action Role Play by enabling the creation of apparently magical or paranormal artifacts [24]. Finally, artists have combined digital media with physical locations, props and players on the city streets to create performances that draw on the everyday world as a theatrical backdrop [3].

While these examples demonstrate the potential of location-based technologies to underpin compelling new experiences, they also raise major challenges, especially with regard to touring them to different locations. At the heart of this challenge is the extensive work involved in adapting an existing experience to each new setting in which it is to be deployed, potentially including authoring content, but also rolling out and tuning the underlying location-service and other technical infrastructure. Although authoring content is a challenge for established digital media such as computer games, location-based experiences raise the added problem of potentially requiring re-authoring for each new setting, with digital assets such as videos, sounds, text, graphics and virtual worlds being created afresh to match local buildings, artifacts, people and other physical assets. Even when previous content is available, it needs to be tailored to fit new locations incurring considerable additional cost. Authoring may even require the creation of props, sets, clothing, and other physical resources that also form part of the content.

After creating assets, the designers have to wrestle with the complexities of location and communication technologies so as to properly locate these within the physical world. Previous studies have shown how challenging this can be, requiring them to be able to decide on the fine details of coverage and accuracy of wireless communications and positioning across a given location [8]. They may even have to actually deploy some new physical infrastructure such as tags and beacons within the new environment. Nor does their work end here, as other studies have highlighted the importance of the real-time monitoring and ongoing orchestration of an experience from behind the scenes once it has gone live [4]. While game-mastering and other forms of orchestration are already practiced with current online games, location-based experiences introduce a new level of challenge, as participants are often widely dispersed across a physical location, difficult to identify, and may suffer from frequent disconnections [3]. 
Finally, these various challenges are mutually dependent, with both the authoring of content and the orchestration of experience depending on the successful adaptation of the underlying technical infrastructure, requiring designers to wrestle with a complex mix of experiential and technical issues at each new location. Unsurprisingly, it remains remarkably difficult to create an experience that benefits from a rich integration of the digital and physical and yet can be easily rolled out across many different locations. Indeed, an inherent lack of 'tourability' may be the greatest bottleneck facing the future widespread adoption of these kinds of emerging location-based experiences.

\section{Studying Rider Spoke}

This paper presents a study of a professional location-based experience called Rider Spoke that has been deliberately designed to tour to multiple cities. Our study covers the critical fifteen-month period from early concept workshops through touring three cities, by which time it had emerged as a stable product. We focus on how artists addressed the complex challenges of tourability by creating a novel structure that, with only minimal seeding, enables participants themselves to create location-based content as they explore a city, while simultaneously driving the gradual evolution of the underlying location-service.

We also describe how the artists iteratively developed new software tools to support the touring process. Our study draws on multiple sources of data, from questionnaires completed by participants that give insights into their experience, to notes and observations from meetings and performances, including ethnographic data gathered by physically following participants and sitting in ranking and filtering sessions. This data brings together in one place both broad and 'thick' descriptions [26] of the activities of artists and technicians, thereby presenting a holistic account of the story of Rider Spoke from multiple perspectives. The first half of our study takes us from creating the initial concept up to the premiere performance of Rider Spoke at London's Barbican centre in October 2007.

\subsection{The design drivers behind Rider Spoke}

Prior to Rider Spoke, our artists had created several location-based experiences and through these, had acquired extensive first hand experience of the substantial costs involved in touring them to different locations. Their primary concern this time was to create an experience that was deeply engaging and yet also lightweight and flexible enough to be toured to many different locations worldwide. Early design workshops explored this challenge and homed in on three key design drivers that would underpin Rider Spoke and reduce the cost of touring. 
A commitment to user-driven locally-relevant content. Participants would populate the experience with content by recording personal stories that others could then listen to. It was important that these stories should be deeply engaging, for example moving, charming or hilarious, but also have local relevance, that is, resonate with specific locations within the city which would somehow serve as an inspiration or backdrop for them. Thus, participants would be required to explore the nooks and crannies of the city in order to record their stories and find those of others.

A lightweight and adaptable approach to location. The location mechanism would need to be sufficiently lightweight and flexible to be reliably deployed and adapted to different cities. Although widely available in principle, the artists' previous experiences led them to reject GPS as being too sensitive to the particular geography of built-up urban environments, especially with regard to variable coverage, and so they were keen to experiment with an alternative approach to location.

Shifting away from a dependency on real-time data connection. Unlike many earlier experiences, Rider Spoke would deliberately operate as a disconnected experience, isolating participants from immediate contact with one another so as to create a calm and contemplative atmosphere, whilst also avoiding the severe technical challenges of dealing with disconnection that had dogged previous experiences and required extensive orchestration.

From these three principles emerged the detailed design of an experience that involves individuals cycling through a city at night, recording and then listening to personal stories that are associated with particular locations. It was felt from the start that travel on foot was too spatially constraining and that some form of transport would be required to enable a sufficiently wide ranging exploration of the city. Cars were briefly considered, but cycles were soon settled on as they allow exploration, can access a wide range of territories, help isolate players and yet still deliver a visceral experience of the city. It was also decided to stage the experience at dusk and into the night so as to heighten the sense of an unusual, even otherworldly, experience of the city as an inspiration for stories. Participants would therefore cycle through the city at night. The artists themselves expressed their intention in their documentation as follows: "As you roll through the streets your focus is outward, looking for good places to hide, speculating about the hiding places of others, becoming completely immersed into this overlaid world as the voices of strangers draw you into a new and unknown place. The streets may be familiar but you've given yourself up to the pleasure of being lost." 


\subsection{A participant's view of Rider Spoke}

Participants arrive at the hosting venue, either on their own bicycle or to borrow one. They register at the reception where they sign a disclaimer and leave a deposit. They then receive a briefing that covers the nature of the experience, how to use the technology, how to cycle safely, and also tells them of an emergency paper map and phone number under their saddle. The receptionist logs their details in a database and then sends them outside where a technician mounts a mobile console (a Nokia N800) onto the handlebar of the cycle and, for loaned cycles, adjusts their seating position so that they feel comfortable and safe. Riders leave the venue individually and the experience lasts for an hour. After the first few minutes cycling, gentle music plays, setting the tone for the experience, and a narrator begins giving instructions. The female voice is calm and measured, adopting a style and tone reminiscent of a psychotherapist:

This is one of those moments when you are on your own, you might feel a little odd at first, a bit selfconscious or a bit awkward. But you're all right and it's OK. You may feel invisible tonight but as you ride this feeling will start to change. Relax, don't forget to breathe both in and out and find somewhere that you like, it might be near a particular building or road junction, it might be near a mark on a wall or a reflection in a window. When you have found somewhere give yourself a name and describe yourself.
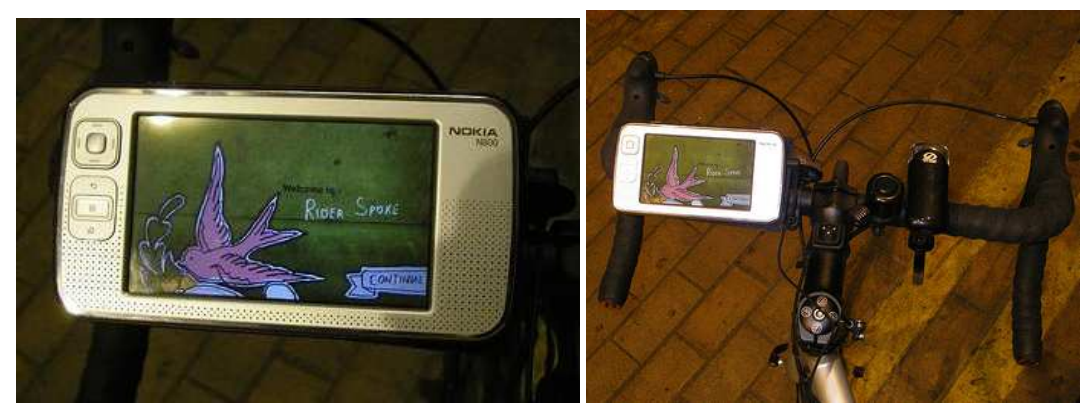

Figure 1. The console mounted on a cycle

The rider's first task is to find an appealing location, stop, and record a name and description. This location has to be a 'new' location within the experience, meaning one that is not already occupied by an existing recording (according to the location technology that we describe below). It is the basic rule of Rider Spoke that each new recording has to be made at a new location, requiring players to continually seek out new places in the city as the volume of recordings grows. When the Rider has found a valid location the console screen displays the invitation shown in figure 2 . 


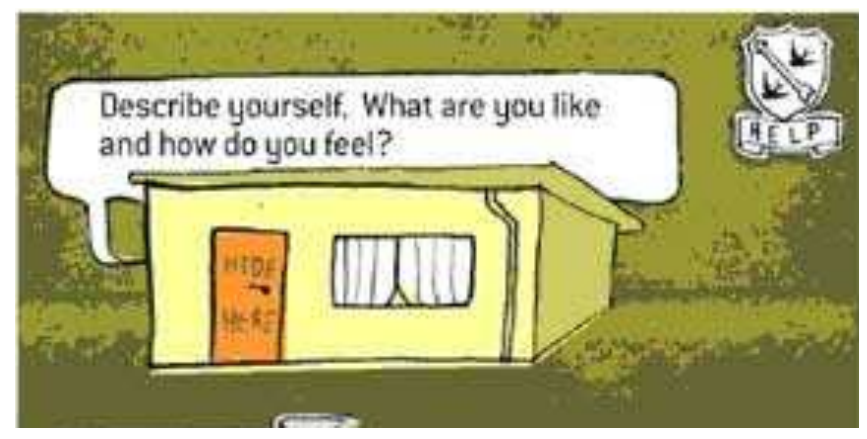

Figure 2: Inviting a rider to record their description

The interface is designed to be distinctive and yet simple, providing just a few options to record and listen to messages. After all, Riders may be using this device for the first time while engaged in an unusual experience that also involves cycling. The overall metaphor is one of hiding and finding recordings within homes. Artistically, the design draws on a combination of Mexican votive art, with religious associations of prayer and offerings, combined with images of tattoos that are emblematic of voyages of exploration. Interaction is via a touch-screen using just a few large buttons and with safety in mind, the briefing and instructions encourage riders to stop before interacting.

Following this initial task, riders can repeatedly choose between recording an answer to a new question or listening to others' recordings, repeating this choice until their allotted time of one hour has elapsed. To answer a question, the rider must again find a new location. They will then be asked a question (over the audio in their headphones) that encourages them to divulge a personal story of some kind. The questions, authored by the artists, ask riders to reflect on significant, evocative or hilarious moments of their life while engaging with the city. For example, they may be asked to reflect on people or events in their lives:

Please will you tell me about your father. You might want to pick a particular time in your father's life or in your life. Freeze that moment and tell me about your dad: what they looked like, how they spoke and what they meant to you. And while you think about this I want you to find a place in the city that your father would like. Once you've found it stop there and record your message about your father at that moment in time. 


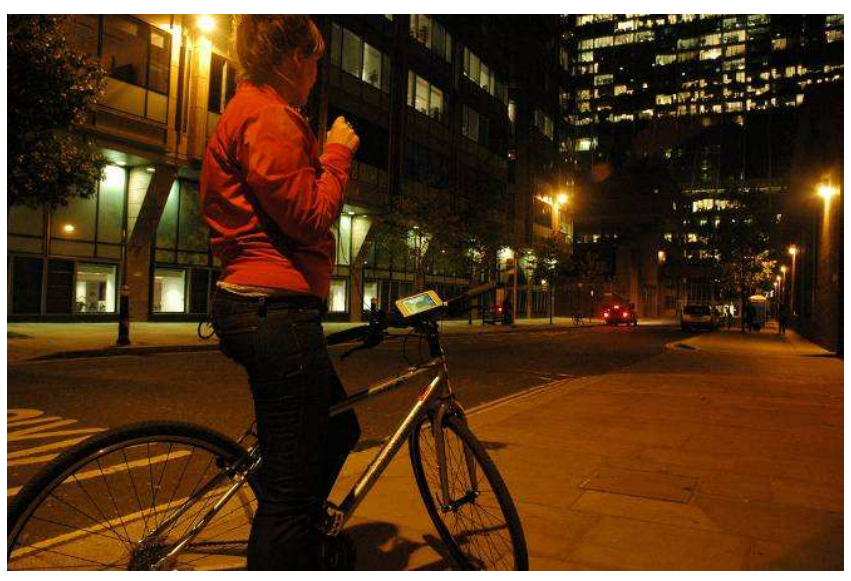

Figure 3. A rider listening to a recording

Or they may be asked to use the places and strangers in the city as inspiration for their imagination: I want you to look for a flat or a house and find a window that you would want to go through. I want you to stare into that window and tell me what you see and tell me why you want to go through that window. Riders are given the opportunity to review and record their stories and then to save them when they are satisfied. On choosing to listen to other's recordings, the rider is offered a list of the three nearest recordings to their current location. As a result, recordings are always available to be heard, although cycling through the city will continually update the list, effectively browsing a landscape of stories. When the experience is near its end the narrator gives the rider one final task: to make and record a final promise. After this, they return to base where the device is dismounted from their bike and their deposit returned.

\subsection{Implementing Rider Spoke}

A substantial portion of our study is concerned with how the artists adapted the technical infrastructure of Rider Spoke, and so it is necessary to delve into some details of its implementation, especially with regard to the design of the location service and database. The design team elected to exploit the prevalence of Wi-Fi in many cities as the basis of their location service as previous research had shown that Wi-Fi could provide a sound basis for a location service [15]. After some initial experimentation, the team elected to develop a bespoke Wi-Fi location service that was tailored to the particular requirements of Rider Spoke, basing this on an existing technique called Wi-Fi fingerprinting [9, 22]. The rider's console periodically scans for Wi-Fi access points as they traverse the city. The resulting list generated at any moment is taken to be a distinctive fingerprint for their current location. If this fingerprint is deemed to be different from already known fingerprints held in a database then it becomes a new location. If it is very similar, it is considered to match an already known location. 
As players record stories, some of these known fingerprints become associated with recordings. The database also records adjacency links between fingerprints that are created whenever one fingerprint is seen directly after another. Thus, the resulting database takes the form of a mathematical graph, with nodes being fingerprints and edges representing direct adjacencies between these. These adjacency links enable the location-service to reason about the relative proximity of locations by working out the minimum number of links that separates them. Thus, it becomes possible to work out the likely 'nearest' recordings to a given location.

Riders begin with a preloaded version of the database on their console from the previous day. Any new locations and data that they generate during the experience as they make recordings and explore new parts of the city are offloaded back to the central database when they return (remembering that this is a disconnected experience). At the end of each day all new locations and new recordings from that day's participants are merged back into the database. This requires the artists to filter and merge the new recordings, working out which should be made available to future riders. This involves listening to all of the recordings from that day and making two key decisions. Firstly, is a recording engaging enough to warrant it being included? Secondly, where two new recordings are associated with the same new location (two riders having independently discovered and used it on that day), which should be kept and which thrown away in order to resolve the conflict? Both of these concerns might be said to be oriented to managing the quantity and quality of content. This in turn reflects an implicit feature of the event: the graph-structured database of known locations, adjacencies and recordings grows and evolves as the experience unfolds over many days. Thus, as it progresses over multiple days in the same location the content is continually being added to and as the quantity of recordings increase so do the number of higher quality recordings. There is a sense, then, in which the work of filtering content is also a job of continual re-calibration, using each new day's recordings as a means of gauging not just their own viability but also the viability of what has gone before. The strength of this approach is that the content and location information evolve from practically nothing as the experience gradually rolls out over the city. A little seed content, normally of the order of a few tens of recordings, and a small fingerprint graph that can be built from a few short test cycle rides around the venue, is all that is required to prepare the experience.

It is important to note that this approach does not deliver the geographical position (coordinates) of a fingerprint; it is therefore not a positioning service, as are some other Wi-Fi-based services. However, it does satisfy the essential requirements of Rider Spoke, namely being able to identify new and existing locations to a mobile console, associating some of these with content, and reasoning about their relative 
proximities. Of course, there is still a question of 'accuracy' in the sense that recordings ideally need to be heard near to any physical locations to which they refer. However, this is deliberately a relatively fuzzy association and so the experience is carefully designed not to rely on accurate positioning. Early seed messages that are deposited by the artists are carefully constructed to be engaging but also locationally vague as these may be potentially heard from far away while the initial graph is small and sparse. As the database grows and the graph becomes ever more dense, it should be the case that the three 'nearest' recordings are increasingly from locations that are actually nearby.

\subsection{Feedback from the premiere performance}

The premiere performance of Rider Spoke took place in London in October 2007, being experienced by 548 riders over 8 days. Between them they left 1,964 audio recordings in the city (average of 3.5 per player and maximum of 10). All riders were invited to give feedback after the event via a web-based questionnaire that probed their background, overall enjoyment, and the themes of recording and listening to stories, finding locations, and cycling. Given that this was a public event, all of the participants were self-selecting, as were the 71 (13\%) of these who completed the questionnaire.

Responses were quite evenly distributed across the 8 days of play, with no day receiving fewer than 5 or more than 10. 41 respondents were female, 28 male, and two didn't say. Most were attracted by the novelty or by the artists' reputation, and some by the cycling (54 classified themselves as 'cultural/arts events visitors' and 29 as 'cyclists'). Over half cycled regularly and 31 used their own bikes. However, 2 claimed to 'never cycle'!

The overall reception to the premiere performance of Rider Spoke was very positive. On a five point Likert scale, 70\% of respondents agreed or agreed strongly that 'taking part in Rider Spoke was fun' while $67 \%$ said that they would do the experience again. While it may of course be that those who felt most positive were also the most willing to complete the questionnaire, this fits well with anecdotal feedback that we received from riders as their returned to the venue. It appears that riders appreciated several aspects of the experience.

They enjoyed recording and listening to stories which could at times be deeply personal and apparently honest, and some even cried while recounting them: "It was a very 'moving' experience for me and very memorable. To be alone in the city, holding your bike, blubbing about your father on a Sunday evening is something else....!" Others, however, invented stories: "I was much more performative. I often made things and characters up to make it interesting, both for me and the other listeners". Listening to other people's stories could also be moving, again especially when they were personal: "One about the 
memory of a man's father. It was extremely touching - he also described standing on a roman wall, an evocative historical location to be thinking about memories. He even vowed to bring his dad to that spot because he thought he'd like it there. Beautiful."

Respondents also remarked on the distinctive social nature of the experience, which as one put it, involved "a disconnected intimacy with a total stranger." This was amplified by the feeling of being isolated in the city: "It was a private moment strangely enough not always in a private space." This is not to say that riders were not aware of bystanders though, which for some introduced a further sensation of being exposed and even slightly vulnerable.

Location played an important role in inspiring stories, with players choosing movie-like backdrops for their recordings: "I just found places that excited me to stand in, that would be seen in a film." and "When hiding in old alleys that reminded me of what Victorian London might have looked like, I think I romanticised the answers a bit more." Ambient sound was also important and would directly appear on the recordings: "Yes - sometimes the background noise or the atmosphere of a particular place really chimed or sometimes jarred with the words I was speaking. This added a layer of richness to the testimonials." Some locations imparted a personal meaning: "I followed a route that I like and stopped at places that had personal resonance or geographic prominence.", while others were simply aesthetically pleasing in their own right: "Cos they were quiet and beautiful in their own way." Yet others were associated with a memorable landmark: "I looked for spots which were quiet and had some kind of marking point - as if it was buried treasure, so like a squirrel I could always find it again if $i$ wanted to!"

Finally, cycling appeared to engender a sense of freedom and liberation in some that may have opened them up to recounting stories. "I love cycling and haven't cycled regularly for a while as my bike broke and $i$ have not got round to fixing it! So it reminded me of the freedom i feel when I cycle and to cycle with no destination is wonderful - I felt physically awakened and engaged" and "Made you look, made you stare, made you investigate dark places you wouldn't normally."

This is not to say that all players had a great time and that all stories were deeply engaging by any means. Many stories were not greatly interesting, others were unintelligible, and there were clearly moments when players found themselves in a completely inappropriate location: "I wasn't going to speak at length about my father whilst standing in a grimy back alley that stank of excrement." Some players got lost, while others commented on being cold, tired, uncomfortable and even in desperate need of the toilet while cycling (see [25] for a discussion of cycle-based interaction informed by Rider Spoke). Perhaps the most frequent criticism, however, arose from the slow response of the interface, 
which could take many seconds to respond on some occasions as we discuss below. However, it does seem that on balance, the distinctive combination of freedom, isolation, vulnerability, disconnected intimacy, location, physicality and of course the artists' questions provided a framework within which many riders could create powerful content.

\subsection{Lessons for the infrastructure}

While the artists were generally satisfied with the overall user experience, the technical challenges involved in delivering Rider Spoke proved to be more problematic. These revolved around two issues: the overhead of filtering content and the somewhat opaque and inflexible nature of the location mechanism.

Given the high profile and often unpredictable nature of a premiere performance, the artists were prepared to devote a large amount of their own time to the hands-on running of the experience, and had also been willing to hire a relatively large crew to support them. This had made it (just about) feasible to listen to and filter hundreds of new recordings each day ready to be merged into the database for the next day's play. However, future touring would need to rely on a smaller crew to be financially viable, and would also have to demand less time from the artists themselves as it would take place over many years to come when they would be working on new projects. Concerns were also raised about how filtering would work with overseas touring where recordings would not necessarily be in English. Improving the filtering of content was therefore identified as a key bottleneck to be addressed to support future touring.

The second issue concerned the location mechanism. Collectively our riders logged 1,236,120 distinct sightings of 3990 unique fingerprints (locations) over the 8 days. The size of the resulting graph was a major contributing factor to the poor performance of the console software. Discussions between the artists and the technical team after the first few days revealed that it was not possible to retune the mechanism to produce a new, smaller graph. Even if it had been, it was unclear what density of graph would be needed anyway. A large and dense graph could clearly cause performance problems and also make it possible to appear to place many recordings at each physical location. Too sparse a graph however might not provide sufficient locations for new content and could potentially lead riders to perceive mismatches between content and location (which had not been a significant factor in user feedback from the premiere). Furthermore, it was not clear how variations in Wi-Fi density across different cities, or perhaps even across a single city, would impact on tuning the graph. Being able to 
tune the fingerprint graph to match the local characteristics of each new city was therefore felt to be also important to the future touring of Rider Spoke.

\section{From Premiere to Touring}

The second part of our study considers the work that was done to address these challenges as Rider Spoke toured through two further cities, Athens in March 2008 and Brighton in May 2008. Nearly all aspects of the experience were tweaked to some extent (questions revised, briefings updated, and the software on the console restructured to improve performance). The event in Brighton was studied in greater depth through the use of an ethnographer physically following a number of cyclists around as they participated and we present some of the relevant findings that came out of that investigation here. The greatest effort, however, was invested in developing tools and processes to support filtering of content and tuning the location mechanism. The work of filtering and handling the location mechanism were also studied in greater depth and these aspects, too, are discussed in more detail here.

\subsection{Being a participant}

As mentioned above, participation in the event was studied in much greater detail during the event at Brighton by means of having an ethnographer trailing willing participants as they rode around the city, collecting and leaving messages. The advantage of capturing in situ engagement in this way was that it enabled us to see what the actual work of participating involved and what kinds of problems arose and how they were handled on the spot. The data was gathered by an ethnographer following each participant on another bicycle at a distance (so as to preserve the individual character of the experience), using a video camera to capture the cyclist's actions, whilst the cyclist was fitted with a recording device and microphone to capture both what they heard and what they said. In addition the cyclist was fitted with a GPS device to keep track of their exact trail through the city. This approach was adopted throughout one whole evening of the event and three cyclists were followed, each ride lasting for in excess of an hour, the last one concluding in the small hours of the morning. This data serves as a rich adjunct in that case to the other data already mentioned above.

Analysis of the ethnographic data was tackled from an ethnomethodological perspective [27]. The focus here is upon explicating how the local interactional order gets produced and how activities are reasoned about and accounted for in the course of their production. The use of this approach to inform design is now firmly established in the systems design community [28]. The following summarizes the principal findings that came out of this analysis: 
Intersubjectivity: One of the things participants in Rider Spoke have to routinely do is orient their activities as both a cyclist and a participant to other people in the city around them. Some of this is about ordinary cycling behaviour: taking care to avoid pedestrians; being duly attentive to traffic, signaling appropriately; etc. Some of this is also about orienting their activities to what is coming to them through their headphones and the handlebar mounted interface from the game, for instance by recognizing and appropriately following requests. However, riders also reason not just about their own activities but about how their activities will themselves be reasoned about by others. This being the case, what Rider Spoke quite particularly does for participants is to raise other considerations that ordinary cyclists would not necessarily face. One such matter is taking into account potential over-hearers. It was manifest that the cyclists understood themselves to be visible and audible to others and they chose places to stop and ways to position themselves accordingly by not stopping in heavily populated spots and by leaning in to the device when recording, rather in the same way as one would when delivering a confidence. This was further accentuated by an orientation to the putative future listeners of their recordings. They did not typically choose spots to record things that were noisy or open to interruption. Thus, the very awareness riders have of their visibility contributes to the goal of seeking out more isolated places appropriate to introspection.

Receiving and following instructions: A core part of Rider Spoke is that the riders are delivered through their headphones particular messages that might be understood to be instructions. Instructions are handled in interactionally distinct ways from other things such as suggestions. Instructions are oriented to as things one should do. Otherwise one should be able to account for not doing them. There is thus a basic orientation to fulfilling what is recognized as an instruction and, by honouring the spirit of what was understood to be, being asked of them through their headphones, it was evident that the riders were orienting to them as instructions. However, what proved to be interesting was the extent to which certain facets of the instruction were routinely ignored, e.g. 'go somewhere you like', 'go somewhere quiet,' etc. Instead the instructions were oriented to as guides for action rather than firm directives. At the same time the riders regularly delivered things that were not explicitly asked for, for instance a description of their location (and, as we will be discussing below, explicit reference to one's location was something that almost always resulted in a response being ranked higher by those doing the filtering). What we can say here, then, is that part of 'playing the game' is to see that being told to do something counts as an instruction - make a promise, keep cycling, tell me about a party, etc. - whilst other aspects such as being told to go somewhere (especially if it was non-specific) to make the promise, etc., do not count as an instruction. Of course, it is hard to obey locational suggestions anyway when one finds when one gets 
there that you cannot leave a message. What is apparent is that fulfilling an instruction means finding somewhere you can stop that bears some relation to what you've been told to do (leave a message; make it a promise; make it about your dad; and so on). The critical observation to make here is that, whilst the participants are instructed in how to proceed through the game, including the generation of future game content, the ways of relevantly following those instructions has to be continually negotiated by the riders into their progress through their immediately surrounding environment. It is the continual job of the riders to figure out 'what will do'. And 'what will do' may or may not have much to do with how the event was conceived by its creators, especially when the riders find they are thwarted in their efforts to leave a message every time they stop.

Licence to be other than an 'ordinary' cyclist: Another aspect of Rider Spoke that moves it beyond being an ordinary cycling experience is the licence it gives to explore the city in ways that would not normally be considered. One cyclist for instance was followed through barriers to the top storey of a multi-storey car park at one o'clock in the morning, where he had ridden expressly to record a message. Another participant cycled through a park in the dark and scrambled up some rocks for a similar purpose, something that might be considered risky on other occasions. A lot of the time the participants clearly do observe the ordinary ways of doing cycling, being amongst people in busy public spaces, and so on, as we discussed previously with regard to matters of intersubjectivity. Where the 'licence' seem to arise is with regard to location and we have already made clear that this is routinely oriented to as the most flexible feature of an instruction. Where it is less evident is with regard to the ways in which participants go about interacting with other people. One message asks the riders to follow someone for a while and comment upon what they look like and what they are doing. All of the participants exhibited varying degrees of reticence about engaging with this instruction and clearly viewed it as potentially accountable in ways that going to unusual locations one would not normally go to on a bike was not. Thus it would appear that there are definite parameters on what degree of licence participation might give. In view of how important the location mechanism is from a technical perspective (as will be discussed again below) the matters of how location is handled in relation to instructions and how Rider Spoke apparently gives licence to participants to toy with understandings of appropriate location are important lessons for future consideration in design.

Intimate encounters with strangers: In the London premiere of Rider Spoke one participant described the experience as being like "a disconnected intimacy with a total stranger." In fact detailed observations and analysis of the actual behaviour of riders during the event at Brighton revealed this to be a particularly apposite comment. At first sight the content coming through the headphones to the 
riders could be oriented to in all sorts of ways. One orientation we have already mentioned is to treat the messages as instructions. Parts of them are also clearly oriented to as suggestions (e.g. with regard to location). Other parts of the content, such as the music played whilst the riders are riding, could be oriented to as ambient noise. However, some of the content that is delivered is recordings of messages left by other people. One immediate orientation to this kind of content, especially as riders encounter it for the first time, is as an object of enquiry and curiosity. However, there is something additional to observe about this. In fact personal messages left by other riders work as a kind of prompt to further confidences by the rider who has been listening to them. As conversational objects in the course of a conversation confidences are not wholly neutral. Instead, confidences turn out to be highly implicative of a further confidence of some kind from the person who has just received one [29]. What happens as riders cycle around is that, even though it is not expressly organized in this fashion, the recordings riders listen to and the recordings they themselves make are something that is experienced as unfolding sequentially. Here's the thing, when you're locked into a sequence of that kind: you don't give a confidence or listen to a confidence and then provide something rigidly formal straight after. Thus the very sequential unfolding of the event is indeed a powerful structure for organizing exactly what the London participant noticed: intimate encounters with strangers. It becomes about swapping confidences with people who are otherwise unknown to you because anything less than another confidence of some kind would be seen as accountable. And, although the riders are in one sense on their own, the earlier observations we made about intersubjectivity make it clear that riders do orient to there being a potential audience for the things they say. The only alternative available is to say nothing at all. But the riders understand there to be a possible audience for their silence as well.

\subsection{Managing the user generated content}

Having discussed in the previous section how the riders themselves orient to and manage the creation of content, we are now going to take a more detailed look at how the artists themselves managed the matter of dealing with content. Filtering recordings needs to address three issues. The first issue is selecting the best content so that it can be merged into the database and made available to future riders. The aim here is to both reduce the volume of content to a manageable amount, ensuring that enough locations remain free for the new players, and also to ensure that that the content is of the best possible quality in the opinion of the artists. The second is the need to resolve conflicts where two new recordings occupy the same location. Thirdly: the challenge of dealing with multiple languages. Many riders in Athens wished to record their stories in Greek and the artists (who did not speak Greek) felt that the recordings 
would be of higher quality if this were the case. To be able to tour Rider Spoke, the artists needed to be able to outsource the task of rating content to trusted people who could be hired in as needed, including native speakers. The first step was development of the following five point rating scale and guidelines as written down in the 'Operator Manual':

1. I can't hear this/would never want to listen to this. Inaudible. Very long silences. Criticizing the work: "This is boring”. Repeated answer: i.e. an exact copy of this answer already exists

2. I wouldn't want to listen to this. Boring: an observation or sentiment that is completely indistinct or obvious "Some parts of the city are quite rough" "I went to lots of wild parties when I was young". These answers lack anything specific, precise or personal. They are often very short. An answer that is almost exactly the same as a previous answer

3. I wouldn't mind listening to this. Average: a typical answer but nevertheless one that has meaning to the person speaking. "I promise that tomorrow I will be kinder to my work colleagues." "I went to a party when I was 15 and tried to kiss a boy called Peter but before I could I threw up ...” The majority of answers will be 3 and, if you are not sure what score to give, mark an answer as 3

4. I would enjoy listening to this. Outstanding. Contains a unique or specific insight or revelation. Something that no one else has said. Makes you laugh or moves you.

5. I would love to listen to this. Exceptional: hilarious, moving, surprising, unique. Not necessarily long: it could be very short

The artists also proposed the following modifiers:

If an answer is location specific (i.e. refers to a specific building or street so that another player would know what they are talking about) +1

Any answer to "describe yourself" (because these are usually short and introductory) -1

Any answer to "make a promise" (because there are so many of them and they can be very repetitive) -1 Very brief (don't forget the effort that a player will have gone to in order to hear an answer: if an answer is very short then it may seem like a big anticlimax unless it is especially strong) -1

These guidelines were then embedded in a web-based ranking tool. One of the screens in this tool presented cases where multiple new recordings conflicted with a single new location. The tool also allowed the artists to choose a ranking threshold (1-5) such that only recordings rated above this would be merged into the database. This could be raised over time as higher quality recordings became available. In each of the three performances the ranking began at 3 on the first day and had been raised 
to 4 by the end (retaining 10\%, 13\% and 20\% of the recordings in London, Athens and Brighton respectively).

The work of ranking was usually shared out among the artists who could review prior rankings day-byday to allow for ongoing artistic oversight and the review of one another's judgments, whilst keeping the task at a level that was practically manageable. Practical management involved the situated use of a variety of shortcuts and workarounds, indexed by the guidelines they had created, e.g. knowing what to ignore completely and what could be decided rapidly. Artists also became much quicker at the task as their familiarity with the content and this new tool grew. Furthermore, the use of the ranking tool and how the above gross categories and rules might get applied was something that itself evolved as the experience became more populated with content and the artists listened to more recordings. Rankings could even be pre-reviewed within the current session to get a feel of what the overall batch of recordings sounded like in advance of the final ranking at the end of the day. In this way ranking sessions and the use of the ranking tool within them became a fine-tuned and highly nuanced resource for keeping content captivating and up to date, with the ongoing work of calibration being applied to the content listened to in the session itself, prior ratings being potentially changed as other content was listened to. The work of calibration also allowed for concerns such as the need for authorial revision. As one of the artists commented: "too many $1 s$ and $2 s$ would indicate a need to reword some of the questions". In addition, a particular attentiveness to locally grounded stories enabled the artists to further refine the location-specific tuning of the experience and imbue the whole event with sensitivity to the character of the locale that it would otherwise have lacked. Finally, being web-based allowed the task to be carried out remotely, further reducing touring costs and allowing the sharing of the work across the team of artists.

\subsection{The complexities of the location mechanism}

Tuning the location mechanism to each new city was the second major challenge for touring Rider Spoke. It also proved to be a considerably more knotty problem to resolve. Given that the artists spent considerable effort trying to understand the technical details of this mechanism, it is necessary for us to briefly consider them here too.

The location mechanism goes through several steps to decide whether the list of currently visible Wi-Fi access points represents an existing or a new fingerprint (and hence location). Wi-Fi scanning software scans every few seconds, returning a list of all currently visible access points, each represented by its MAC address (the unique address pre-programmed into the access point) along with the received signal 
strength with which it has been seen. Access points below a configurable signal strength threshold, are then removed from the list because they may be susceptible to transient interference and their inclusion could lead to highly unstable fingerprints. After this, only a limited number of the remaining access points are considered, according to a configurable fingerprint size parameter, with the weakest signals being discarded. The result of applying these two parameters is a manageably long list of access points, each of which is associated with reasonable signal strength. The resulting list is then compared against the local database of known fingerprints (the fingerprint graph). If the filtered access point list "overlaps" an existing fingerprint by a sufficient amount - specified by a third overlap threshold parameter - then the new sighting is deemed to match an existing fingerprint. If not, it is deemed to be a new fingerprint and is added to the fingerprint graph along with a new edge that connects it to the most recently sighted fingerprint before this. The overlap is the proportion of access points that are in common between the two lists, for example "A,B,C" and "A,B,C,D” have a 75\% overlap. Choosing a value of $100 \%$ for the overlap threshold would require that access point-lists match exactly. In this case any difference in the list would produce a new fingerprint and hence a new location. Conversely, smaller values allow an existing location to be found again if a subset of the access points are not visible, for example if they have been temporarily turned off.

In summary, the three key parameters of signal strength threshold, fingerprint size, and overlap threshold control how raw lists of access points and signal strengths are mapped onto distinct fingerprints and hence locations. Collectively, they determine the density and stability of the resulting fingerprint graph. The artists can change them to produce, on the one hand, a graph with very many but possibly transient locations, and on the other, a graph with relatively few but more stable locations. The goal is to find the correct combination that produces enough locations for a given city so that riders can find new locations at which to place their stories, and yet not so many that it feels like too many stories are at each location or that it is difficult to ever find a story again after it has been recorded.

Discussions with the artists revealed that it was difficult both to understand and predict the effect of changing individual parameters (not least because they overlap to some extent in their ultimate effect). Setting them for the London performance had been largely a matter of guesswork and the result had clearly not been optimal. It also seemed highly likely that these parameters would need to be tuned to suit the particular local distribution of Wi-Fi access points across each new city so that a more principled approach was clearly needed. 


\subsection{Tools to adapt the location-mechanism}

In response, the team created a suite of three software tools to help localise Rider Spoke to each new city. The survey tool was used to gather some initial Wi-Fi data from the city, along with GPS data that could subsequently help geo-reference this to maps of the area. The simulation tool processed this data, enabling the artists to create very rapidly many different graphs, arising from different parameter settings, and to rank and compare. Finally, the visualisation tool revealed the likely distribution of a given fingerprint graph across the chosen area of the city.

The survey tool required someone to cycle several pre-planned routes through the city with a mobile logging device to cover likely places that riders might visit during the experience. The artists aimed to get a broad-brush picture of the environment in as short a time as possible. The Athens survey was conducted by four artists in parallel and completed in about one hour. Here, the different routes were arranged so that they would cover different areas, but also contain some overlap with other routes to make connections in the fingerprint graph. The Brighton survey covered the busy city centre, the suburbs and the seafront and was completed by a single artist in about one hour. These surveys were carried out weeks in advance of the performance alongside the usual site inspection and discussions with the hosting venue and the survey data was then taken back to the studio for analysis.

The simulator tool allowed the artists to conduct a series of 'what if' experiments by feeding the survey data into the fingerprinting mechanism as if live and then stepping through ranges of values for each of the three parameters in order to automatically generate hundreds or even thousands of different fingerprint graphs. These graphs could then be sorted according to basic metrics including the number of fingerprints and edges they contained. An additional metric called 'retrievability' was introduced to capture the artists' requirement that "content and fingerprints must be easy to find". This was defined to be the total number of fingerprint sightings divided by the number of unique fingerprints generated for this graph and helped the artists find parameter settings that yield a graph whose locations might be suitably easy to find. By sorting and sifting to compare these various metrics across many graphs, the artists were able to home in on parameter settings that might be appropriate to a good number of locations that were reasonably retrievable.

Finally, the visualisation tool enabled the artists to inspect selected graphs (chosen from the simulation), examining their topologies (the clustering and connectedness of fingerprints) in relation to the geography of the local area. The graphs could be drawn as abstract visualisations to reveal their topology, but these could also be pinned on to various geographical maps and satellite views of the local area in Google Earth. This pinning used the GPS data captured alongside the Wi-Fi data to position 
some of the fingerprints on the map and then stretched the remaining (non-GPS) fingerprints out between them by treating their edges as springs. As an example, figure 4 shows a geo-referenced visualization of a graph containing 120 fingerprint locations, which were chosen from the simulation tool and then overlaid on a map of Brighton. Although the graph is small when compared to a final graph, the route has been carefully chosen to cover several typical terrains within the city including the busy city centre, quieter suburbs and the seafront. This distribution of locations looked fine at first sight, with fingerprints being generated along all parts of the route. However, on closer inspection, it became apparent that there is a cluster of fingerprints in the city centre, where dozens of fingerprints map onto a single real location. Figure 5 shows a zoomed-in view of this cluster overlaid on an aerial photograph.

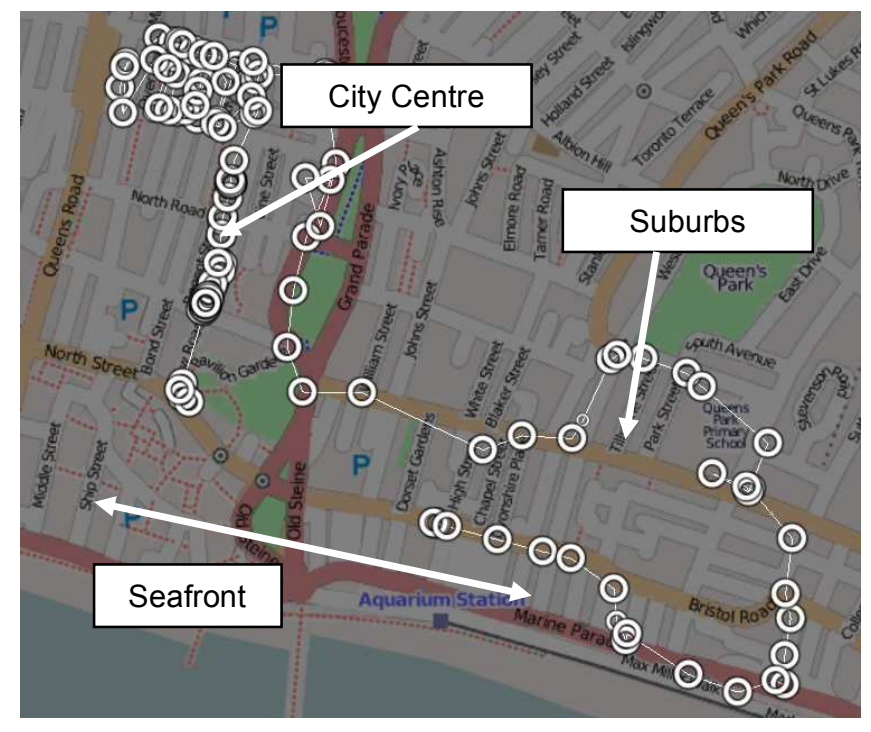

Figure 4. Geo-referenced visualization of Brighton survey

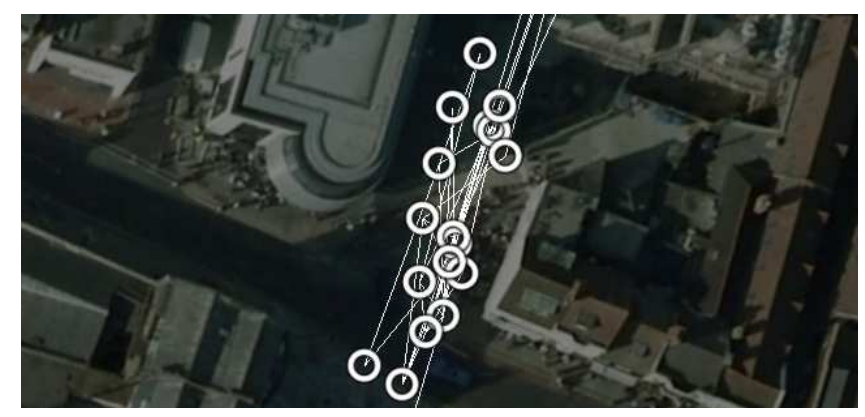

Figure 5. Many fingerprints at a single real location

This cluster was at a street corner where the artist took several minutes to 'kit up' for the survey, but while running the surveying tool throughout. It appeared that with the chosen settings, simply stopping in a busy neighbourhood would generate multiple virtual locations. This was not acceptable to the artists. Trying to find a specific virtual location on this street corner would be very difficult and it was likely the graph would grow to an unmanageable size during an actual performance. Moreover, the 
starting point of the experience was just $100 \mathrm{~m}$ to the north of this street corner, so that many riders would travel through this spot, potentially creating even more fingerprints. As a result, the artists went back to the simulation tool and managed to find different parameter settings that would reduce this problem while still appearing to generate sufficient locations in the suburbs and at the seafront. These were used for the final performance and it ran very successfully.

\section{Populating the City with Stories}

In its varied detail, our study has revealed how Rider Spoke combines a distinctive mode of interaction (cycling through a city at night and recording and listening to personal stories) with a novel technical infrastructure (an evolving graph of locations determined through Wi-Fi fingerprinting) to create a unique experience. The ultimate success of this approach is perhaps best demonstrated by the continued popularity of Rider Spoke as a touring product. At the time of writing it has been performed in London, Athens, Brighton, Budapest, Adelaide, Sydney, Copenhagen, Bristol, Edinburgh and Linz, with further bookings pending. That Rider Spoke is engaging for audiences (and promoters) and yet also tourable can be largely attributed to the way in which both experiential and technical issues have been addressed in tandem through a process of iterative design and testing 'in the wild' of public performance. However, beyond documenting a 'unique' experience, what more general lessons can we learn for HCI? What can the designers of other location-based experiences and technologies take away from this study?

The co-evolution of content and infrastructure. As already mentioned, the critical feature of Rider Spoke is the way in which both the content and the underlying location database evolve in tandem over the lifetime of the experience as a result of participants exploring the city. This enables the experience to be rolled out quickly across each new city without the need for extensive pre-authoring of content, or surveying or deploying underlying infrastructure, directly addressing two major bottlenecks to touring location-based experiences.

User generated content. Web 2.0 services such as Wikipedia, YouTube and others make use of friendships, subscriptions and users' ratings to motivate 'user generated content' and ensure that the most interesting content becomes the most publicly visible (see [14] for a discussion of collaborative editing in Wikipedia for example). Rider Spoke also relies on user-generated content, but in a different way. First, it provides a distinctive framing for storytelling in which members of the public use 
anonymity and isolation, rather than friendship and discussion, to encourage powerful performances. We discussed in our detailed observations above how situated orientations to matters such as instructions, licence and the swapping of confidences contribute enormously to how these kinds of matters pan out. Second, the artists retain a high degree of editorial control over the final rating and filtering of content that is published back to the users (although it has been an active debate among the artists as to whether there should also be a lightweight mechanism to allow riders to highlight particularly moving stories, but without introducing a sense of competitiveness or overt rating that might fatally disrupt the framing of the experience). While having the artists rate content clearly limits the scalability of Rider Spoke (while ensuring high quality), it may be that other applications that employ user generated content can learn from its approach to carefully framing storytelling, to enable the public to perform.

A 'placeful' experience. As a location-based experience, Rider Spoke is also able to draw on specific places in the city to provide rich and often personally meaningful backdrops for stories. Furthermore, participants are required to explore the city in order to find and create these locations for themselves rather than having the artists choose or create them in advance. In other words, in a location-based experience, creating locations can be as much a part of user generated content as recording audio or video. Rider Spoke is also distinctive in that it does not fix locations to absolute positions or demand a high degree of accuracy. One way to view its deliberately fuzzy approach is, as Harrison and Dourish have previously argued [13], that it emphasises a sense of place rather than a consideration of the absolute geometry of the space of the city, which would have arisen from adopting a more positioningoriented approach. However, as Dourish suggests also when subsequently revisiting this argument [11], the division between place and space is sometimes less clear. We would suggest that what we see in Rider Spoke is the development and deployment of a technology that presents a 'user generated spatiality' to users with "people creating space through their movement" [11] as they cycle through the city.

Tools for flexible and adaptable location services. Finally, our study has also shown that evolution of the infrastructure requires careful management in much the same way as the user driven evolution of content. To be effective, this user driven infrastructure needs to be adapted to local conditions (in our case to the local geography and variations in the distribution of Wi-Fi) and this requires careful attention and the support of dedicated tools that enable designers to survey, simulate and visualize the likely evolution of an infrastructure across a particular city and to be able to tune it as a result. This capability 
to reveal the nature of the technical infrastructure to the designers of location-aware experiences reflects an ongoing discussion of 'seamful design' within HCI. Previous papers have observed how the characteristics of the underlying infrastructure of positioning and wireless communications can have a major impact on the user experience and how, far from being an occasional glitch or bug, this is an ongoing aspect of such experiences that needs to be recognized by designers [8]. Lack of coverage or accuracy can be characterized as 'seams' in the infrastructure, a natural part of the fabric from which it is knitted together. Various approaches to dealing with seams have been proposed including removing, hiding, managing and even exploiting them [4]. Work on seamful games has explored the latter approach, demonstrating and studying a series of examples in which partial coverage of Wi-Fi and/or GPS becomes a resource in the game that can be exploited by players, for example, by being able to hide in the 'GPS shadows' [2]. Other work has explored the approach of revealing the infrastructure, arguing that authoring tools that allow designers to overlay digital assets onto a map or image of a physical location need to be extended with an additional infrastructure visualization layer that enable designers to take account of the availability of the underlying infrastructure across specific locations [20]. Our study has demonstrated the power of this latter approach, providing our artists with a series of tools for exposing the characteristics of its location mechanism across each new city. Part of this certainly involves overlaying visualizations of the infrastructure on maps as we see in figures 5 and 6 . However, it also requires additional tools for surveying each site and for experimenting with various settings of parameters in order to explore different possible scenarios. In short, while we agree that designers do indeed need to visualize the underlying infrastructure, we recognize that this is only one part of a complex overall workflow that also involves gathering and experimenting with data and that all of these stages require support from dedicated tools.

\section{Conclusion}

Our study of a touring location-based experience, from its first concept to its emergence as a stable product following three performances in different cities, has revealed how professional artists worked to create an experience that was both compelling and yet also practicably tourable to different locations. We have seen how they developed a distinctive approach that involved participants exploring a city on bicycles, recording and listening to personal stories, which were then filtered and merged together into an evolving database of 'user generated content'. We have also seen how the iterative development and use of survey, simulation and visualization tools allowed this to be supported by a location service that itself gradually evolved at each city and could be tuned to its local characteristics. 
While Rider Spoke itself is perhaps a unique experience, we have also discussed how it embodies general principles that should be of use to the designers of future location-based services. Careful framing of the experience, drawing upon cycling as a distinctive mode of engagement with the city and deliberately isolating participants from one another, while unusual in traditional HCI terms, establishes a frame within which the public are able to create powerful user generated content. At the same time, the development of an evolving platform that can be easily rolled out over a city with only minimal seed content, supported by dedicated tools that enable adaptation to each new setting, enables the experience to be easily toured. It is through attention to detail of both content and technology that the artists are able to deliver a successful experience.

In summary, Rider Spoke shows through its success as a touring product that there are solid, practical ways of addressing some of the core challenges that face the widespread adoption of location-based applications. Indeed, a concatenation of practical, locally-sensible strategies appears to be an inevitable aspect of managing touring location-based services where it is exactly the distinctiveness of particular locales that may trump any effort to create overly generic, standardized solutions. We hope that through the detailed study of artistic practice presented in this paper we have begun to show how some of these strategies might appear.

\section{References}

1. Abowd, G., Atkeson, C., Hong, J., Long, S., Kooper, R., Pinkerton, M., 'Cyberguide: A mobile context-aware tour guide', Wireless networks, 3 (5), 1997, Springer.

2. Barkhuus, L., Chalmers, M., Tennent, P., Hall, M., Bell, M., Sherwood, S., Brown, B., "Picking Pockets on the Lawn: The Development of Tactics and Strategies in a Mobile Game”, Ubicomp،05, 358-374, Springer LNCS

3. Benford, S., Crabtree, A., Reeves, S., Sheridan, J., Dix, A., Flintham, M., Drozd, A., 'The Frame of the game: the opportunities and risks of staging digital experiences in public settings', CHI 2006, 427-436, 2006, ACM.

4. Benford, S., Crabtree, A., Flintham, M., Drozd, A., Anastasi, R., Paxton, M., Tandavanitj, N., Adams, M., Row Farr, J., 'Can You See Me Now?', ACM Trans. Computer Human Interaction, 13 (1), 100-133, 2006.

5. Benford, S., Rowland, D., Flintham, M., Drozd, A., Hull, R., Reid, J., Morrison, J., Facer, K., 'Life on the Edge: Supporting Collaboration in Location-based Experiences', CHI 2005, Portland, USA, 721-730, ACM.

6. Boyd Davis, S., Moar, M., Jacobs, R.,Watkins M., Riddoch, C., Cooke, K., 'Ere Be Dragons: heartfelt gaming', Digital Creativity, 17 (3), 157-162, 2006, Routledge.

7. Brown, B., Chalmers, C., Bell, M., Hall, M., MacColl, I., Rudman, P., 'Sharing the square: Collaborative Leisure in the City Streets', ECSCW'05, 427-447, Paris, Springer.

8. Chalmers, M., Galani, A., 'Seamful interweaving: heterogeneity in the theory and design of interactive systems', DIS '04, 243-252, Cambridge, MA, ACM. 
9. Cheng, Y., Chawathe, Y., LaMarca, A., J. Krumm, J., 'Accuracy Characterization for Metropolitan-scale Wi-Fi Localization', MobiSys, Seattle, Washington, 2005.

10. Cheok, A., Goh, K., Liu, W., Farbiz, F., Fong, S., Teo, S., Li, Y., Yang, X., 'Human Pacman: a mobile, wide-area entertainment system based on physical, social, and ubiquitous computing', Personal and Ubiquitous Computing, 8 (2), May 2004.

11. Cheverst, K., Davies, N., Mitchell, K., Friday, A., Efstratiou, E., 'Developing a context-aware electronic tourist guide: some issues and experiences', CHI 2000, 17-24, 2000, ACM

12. Dourish, P. 2006. 'Re-Space-ing Place: Place and Space Ten Years On', CSCW 2006, 299-308, Banff, ACM.

13. Harrison, S. and Dourish, P. 'Re-Place-ing Space: The Roles of Space and Place in Collaborative Systems'. $C S C W$ '96, Boston, MA, 67-76, ACM.

14. Kittur, A., Suh, B., Pendleton, B. A., and Chi, E. H. He says, she says: conflict and coordination in Wikipedia, CHI'07. 453-462, ACM.

15. LaMarca, A., et al.: Place Lab: Device Positioning Using Radio Beacons in the Wild. In: Proc. Pervasive Computing (2005) 116-133.

16. Lindt, I., Ohlenburg, J., Pankoke-Babatz, U., Ghellal, S., 'A report on the crossmedia game epidemic menace', Computers and Entertainment, 5 (1), Jan 2007, 8.

17. Mitchell, K., McCaffery, D., Metaxas, G., Finney, J., Schmid, S., Scott, A., 'Six in the city: introducing Real Tournament - a mobile IPv6 based context-aware multiplayer game' 2nd Workshop on Network and System Support for Games, 91 100, CA, USA, 2003.

18. O’Hara, K., 'Understanding geocaching practices and motivations', CHI 2008, 1177-1186, Florence, ACM.

19. O’Hara, K., Kindberg, T., Glancy, M., Baptista, L., Sukumaran, B., Kahana, G., Rowbotham, J., 'Collecting and Sharing Location-based Content on Mobile Phones in a Zoo Visitor Experience', Computer Supported Cooperative Work, 16 , 11 44, April 2007, Springer

20. Oppermann, L., Broll, G., Capra. M., Benford, S., 'Extending Authoring Tools for Locaion-Aware Applications with an Information Visualisation Layer', Ubicomp 2006, Organge County, Sept 2006, Springer

21. Piekarski, W. and Thomas, B., 'ARQuake: The outdoors augmented reality system', Communications of the ACM, 45, 1 (Jan. 2002), 36-38, ACM

22. Rekimoto, J., Miyaki, T., Ishizawa, T.: LifeTag: WiFi-based Continuous Location Logging for Life Pattern Analysis. Proc. International Symposium on Location- and Context-Awareness (LOCA), 2007.

23. Rogers, Y., Price, S., Fitzpatrick, G., Fleck, R., Harris, E., Smith, H., Randell, C., Muller, H., O'Malley, C., Stanton, D., Thompson, M., \& Weal, M., 'Ambient wood: designing new forms of digital augmentation for learning outdoors', IDC 2004, 3-10, June 1-3, Maryland.

24. Waern, A., Montola, M., Stenros, J. 'The Three-sixty Illusion: Designing for Immerion in Pervasive Games', CHI 2009, 1549-1558, Boston, MA, 2009, ACM.

25. Rowland, D., Flintham, M., Oppermann, L., Marshall, J., Chamberlain, A., Koleva, B., Benford, S \& Perez, C.

'Ubikequitous Computing: Designing Interactive Experiences for Cyclists'. Mobile HCI 09. 15-18 September 2009, Bonn, Germany.

26. Ryle, G. (1971) Collected Papers, 2, London: Hutchinson, pp. 480-496 
27. Garfinkel, H (1967) Studies in Ethnomethodology, New York: Prentice-Hall

28. Crabtree, A. (2003) Designing Collaborative Systems: A Practical Guide to Ethnography. New York: Springer-Verlag 29. Sacks, H. (1992) Lectures in Conversation, Volume II, Winter 1971, March 4 "Produced similarities in first and second stories: Poetics, 'Fragile Stories’, etc.”, edited by G. Jefferson, London: Blackwell. 